\title{
Vier Fachleute - Vier Behandlungsstrategien Schulter-Arm-Syndrom
}

\section{Mit gezielten Griffen und Injektionen behandeln}

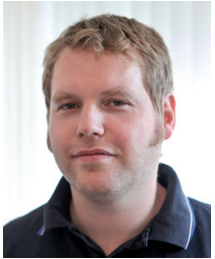

Einem Schulter-Arm-Syndrom können Traumata, Fehlbelastungen, degenerative Schultergelenkveränderungen, ein HWS-Syndrom oder myofasziale bzw. viszerale Dysfunktionen zugrunde liegen. In der Chiropraktik behandeln wir insbesondere die parietalen Dysfunktionen, die zu einer SchulterArm-Problematik führen können. Neben den segmentbezogenen Schmerzauslösern aus dem Bereich der HWS (insbesondere Blockaden im Segment C 4/5 und im zervikothorakalen Übergang) können Ursachen für parietale Dysfunktionen sein:

- Blockaden der Klavikulargelenke (Akromioklavikulargelenk/ Sternoklavikulargelenk [SCG])

- Adhäsionen im Schultergelenk

- Hochstand der 1. Rippe

- Verengung der Scalenuslücke (Plexus brachialis)

- Hypertension M. pectoralis minor (Plexus brachialis)

- Hypertension M. latissimus (Innenrotation Humoralgelenk, ISG auf Blockade prüfen)

Kontraindikationen für eine chiropraktische Behandlung sind: Fraktur, Tumoren, Entzündung, Infektion, Durchblutungsstörung, Bandscheibenprolaps bzw. -protrusion.

\section{Behandlungskonzept}

Liegt ein Komplex parietaler Dysfunktionen vor (Diagnose: Blockade im Segment C 4/5, C 7/Th 1, Dysfunktion im Schultergelenk, Restriktion der Klavikula im SCG, Blockade des Iliosakralgelenks [ISG]) wird nach dem Ausschluss von Kontraindikationen am Achsenorgan (WS) mit den Manipulationen begonnen. Mit Impulstechniken werden die blockierten WS-Segmente der HWS und das ISG sowie das SCG befreit. Anschließend wird das Schultergelenk nach dorsal und kranial mobilisiert. Die Dysfunktion im Schultergelenk wird mit der Hebeltechnik über die Achselhöhle korrigiert. Unterstützend kann mit neuraltherapeutischen Techniken behandelt werden. Die Behandlung erfolgt 1-mal wöchentlich und kann bei abklingender Symptomatik in zeitlich größeren Abständen erfolgen.

- Segmentale Infiltration an den WS-Segmenten und am ISG:

Lactopurum $^{\circledR} 5 \mathrm{ml}$ i.m. + Ledum ${ }^{\circledR} 2 \mathrm{ml}$ i.m. (beides Fa. Pflüger) oder Hanomyloticum $5 \mathrm{ml}$ i.m. (Fa. Hanosan)

- Quaddelung über dem Schultergelenk mit einer Mischinjektion: Procain 2 \% 2 ml i.c. + Chiroplexan ${ }^{\circledR}$ i.c. (Fa. Pflüger) in einer Reihe von der hinteren Axillarfalte über das Akromioklavikulargelenk bis zur vorderen Axillarfalte

n Infiltration der Muskelansätze am Tuberculum majus (Triggerpunkt aufsuchen) mit z. B. Infi Para $\mathrm{H}^{\otimes} 5 \mathrm{ml}$ (Fa. Infirmarius) + Lymphdiaral $^{\circledR} 2 \mathrm{ml}$ (Fa. Pascoe)

\section{HP Christian Blumbach}

Sterkrader Straße 10, 47166 Duisburg; E-Mail: info@blumbach.de

\section{Qi und Blut bewegen}

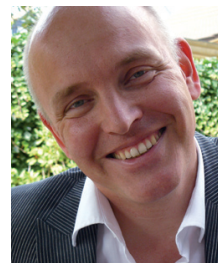

Das Schulter-Arm-Syndrom (synonym: Zervikobrachialsyndrom) lässt sich mithilfe der TCM oftmals gut behandeln. Qi- und Blut-Blockaden über den Meridianen und tendino-muskulären Begleitmeridianen verursachen die Symptome. Aus westlicher Sicht entstehen Symptome im Bereich des Halses, des Schultergürtels und der Arme infolge von Irritationen des peripheren Nervensystems (PNS), Veränderungen der HWS lösen diese aus. Das PNS folgt einer primär segmentalen Gliederung, die Spinalnerven treten jeweils zwischen den Wirbelkörpern aus. Diese segmentale Innervation nutzen wir und behandeln über der HWS lokal.

\section{Stechen, Schaben und Kleben}

Zunächst erfolgt die Akupunkturbehandlung lokal im Bereich der HWS über Du Mai und Blasenmeridian sowie an den HuatuojiajiPunkten. Zudem werden Punkte im dysästhetischen Areal selbst sowie die reaktiven Dü- und Gb-Punkte des Nackens akupunktiert. Die auf den Armen lokalisierten Fernpunkte der Meridiane, die das schmerzhafte Areal kreuzen, werden ebenfalls genadelt. In jedem Fall werden Dü 3 und Bl 62 (Kardinalpunkte Du Mai/Wirbelsäule) SJ 5, Lu 7, Dü 7 und BL 58 (Luo-Punkte) akupunktiert.

Die Gua-Sha-Massage ist eine tiefe Bindegewebsmassage. Die SchabeTechnik (Gua = „schaben“) wirkt bei Anwendung an Nacken und oberem Rücken (Oberer Erwärmer, Bl 10-15) durchblutungsfördernd und sympathikolytisch auf Derma-, Sklero- und Myotom direkt; indirekt auf die Organe und im gesamten Areal der geschabten Segmentebenen. Der TCM zufolge bewegt Gua-Sha Qi und Blut (effektive Therapie der aktiven muskulären Triggerpunkte) und leitet auch Pathogene aus: Ein Zervikobrachialsyndrom wird eben oft durch Wind, Kälte und Nässe getriggert, das Taiyang-Gebiet hier ist anfällig für diese exogenen Pathogene.

Das (Aku-)Tape wird als HWS-Tape geklebt. Durch die unterschiedlichen Elastizitätskoeffizienten von Tape und Haut kommt es durch Bewegungen des Patienten auch zu Scherbewegungen in die Tiefe. Die Effekte sind ähnlich denen der Gua-Sha-Massage. Tapes werden zudem über den betroffen Meridianen der Arme über die schmerzhaften Areale, aber auch kontralateral geklebt.

\section{Behandlungsdauer}

Ich akupunktiere 1-2-mal pro Woche und wende die Gua-ShaMassage an, wenn das durch sie verursachte Sha („Rötung“) wieder abgeblasst ist. Aku-Tapes, geklebt im Anschluss an die Behandlung, verbleiben ca. 2-3 Tage. Oft reichen 10-15 Sitzungen, die Beschwerden schwinden oft von peripher nach zentral. 


\section{Traditionelle abendländische Behandlungs- strategien}

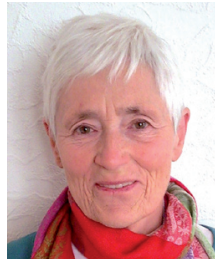

Der Schulter- und Nackenbereich symbolisiert das Tragen und die aufrechte Haltung ebenso wie die Beweglichkeit der oberen Extremität - im Physiologischen und im Psychosomatischen. Die Behandlungsstrategien sollten diesen Bedeutungen folgen. Zudem kann es einerseits wichtig sein, eine energetische Entlastung zu schaffen; und andererseits, bei Energiemangel, Energie zuzuführen.

\section{Therapiestrategien}

Bei leichten akuten oder chronischen Beschwerden, z. B. durch Überanstrengung, helfen Meerrettichblätterauflagen: Pro Schulter ein großes Blatt ohne Blattrippe zerknautschen und über die Schulterkugel bis zum Halsansatz legen, ein enges T-Shirt oder ein Spenzer halten den „Verband“ ohne weitere Fixierung. Schwefelhaltige Inhaltsstoffe, Chlorophyll und die Blattkühle führen schnell zur Linderung. Nach ca. $8 \mathrm{~h}$ kann das trockene Blatt abgestreift werden. Die Behandlung kann bei Bedarf an mehreren Tagen 2-mal tgl. wiederholt werden. Stauungen und Verhärtungen lassen sich mithilfe von Blutegeln gut behandeln. Dazu im Bereich der Trapeziusmuskulatur 2 Blutegel beidseits ansetzen und bis zum Abfallen saugen lassen. Besonders günstig ist es, wenn die Nachblutung lange anhält, das führt zur Entstauung des gesamten Schulter-Hals-Kopf-Bereichs! Ebenso eignet sich das Schröpfen (blutig oder unblutig), es ist weniger aufwendig und wirkt entstauend (blutig) bzw. energiezuführend (unblutig). In jedem Fall bringt es große Erleichterung. Das Gefühl hält häufig über Tage an und hat einen befreienden psychischen Effekt. Über die entsprechenden Reflexzonen können zusätzlich Organzonen, z. B. Tonsillen- oder Lungenbereiche, behandelt werden.

Bei Schultersteife empfiehlt es sich, eine Immunmodulation über den Darm durchzuführen: Fortakehl ${ }^{\circledR}$ D 5 und Pefrakehl ${ }^{\circledR}$ D 5 je 5-8 Tr. tgl. für mindestens 14 Tage (besser 4 Wochen) sowie Citrokehl ${ }^{\circledR} 1 \times 1$ Tbl. tgl. für 6 Wochen (alle Fa. Sanum). Der Schulterbereich sollte örtlich entsäuert werden mit alkalischen Auflagen: 2-mal tgl. 1 TL Alkala ${ }^{\circledR} \mathrm{N}$ (Fa. Sanum) in $200 \mathrm{ml}$ recht warmem Wasser auflösen, ein Tuch damit tränken und für 1/2-1 h auflegen; bei Bedarf auch 3- bis 4-mal tgl. wiederholen. Alternativ sind warme Heilerdeumschläge (Fa. Luvos) möglich. Dazu 2 EL Heilerde mit etwas warmem Wasser breiig rühren, für 2-4 h auflegen und sehr warm halten. Zusätzlich je 1 Amp. Nigersan ${ }^{\circledast}$ D 5 bzw. D 6 und Notakehl ${ }^{\circledR}$ D 5 bzw. D 6 (je nach Testung) (beide Fa. Sanum) alle 3-4 Tage an den schmerzhaften Stellen s. c. injizieren. Außerdem rechtsdrehende Milchsäure RMS Asconex ${ }^{\circledR}$ (Fa. Asconex) $2 \times 30$ Tr. über 3-4 Wochen oral verabreichen. Passive Schulterbewegung durch die gesunde Hand oder über eine Art Flaschenzug/,feste Rolle“ (offene Tür) unterstützen die Lockerung.

\section{Ausleiten mit der Phytotherapie und dem Pflaumenblütenhämmerchen}

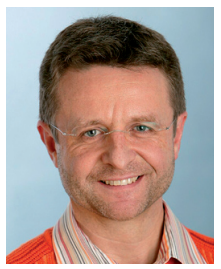

Bei der Behandlung chronifizierter Gelenkentzündungen werden Heilpflanzen eingesetzt, die auf die Gelenkkapseln und das umliegende Bindegewebe einwirken. Sie sollten die Zirkulation verbessern, insbesondere den Lymphabfluss, das Gewebe entsäuern, soweit möglich Ablagerungen in Lösung bringen und einen entzündungswidrigen Effekt haben. Letzteres ist v. a. von Pflanzen zu erwarten, die auf den Prostaglandinstoffwechsel einwirken wie die Nachtkerze (Oenothera biennis) oder das Mädesüß (Filipendula ulmaria). Sauerdorngewächse wie die Berberitze (Berberis vulgaris) oder der Blaue Hahnenfuß (Caulophyllum thalictroides) leiten über Leber und Niere aus und regulieren den Säure-Basen-Haushalt im Gewebe. Ähnlich wirken die Große Brennnessel (Urtica dioica) und die Herbstzeitlose (Colchicum autumnale), wobei diese noch stark antiphlogistische Effekte zeigt. Die Weinraute (Ruta graveolens) und die Rosskastanie (Aesculus hippocastanum) lösen Blutstauungen und wirken abschwellend. Besonders günstige Effekte zeigen Individualmischungen spagyrischer Heilpflanzen.

\section{Beispiel für eine spagyrische Individualmischung} Berberis, Urtica, Ruta, Filipendula, Oenothera, Colchicum ana ad $30.0 \mathrm{ml}$ (Fa. Spagyros). M.D.S.: 3 × 2 Hübe auf die Zunge für 6 Wochen

\section{Beispiele für phytotherapeutische Fertigarzneien}

Heweberberol $^{\circledR}$ (Fa. Hevert) $3 \times$ tgl. 20 Tr. oder Infiossan ${ }^{\circledR}$ Tropfen (Fa. Infirmarius) $3 \times \operatorname{tgl}$. 10 Tr. für 6 Wochen

\section{Hyperämisierende Begleitung}

Begleitend erfolgt eine ausleitende und hyperämisierende Behandlung, z. B. mit dem Pflaumenblütenhämmerchen. Mit diesem 2-mal wöchentlich über dem erkrankten Gelenk sowie über Lunge, Leber, Milz und Nieren an den Rücken-Zustimmungspunkten (Bl 13, Bl 18, Bl 20 und Bl 23) ausleiten. Anschließend mit Rosmarinöl oder -salbe einreiben, z. B. NeproSport ${ }^{\circledR}$ Grün (Fa. Nestmann).

Dr. med. Harald Kämper

Nordring 28, 45894 Gelsenkirchen/Buer 